\title{
Using Superelements in the Calculation of Lattice-Boom Cranes
}

\author{
DR.-ING. MiCHAEL KLEEBERGER, \\ DIPL.-ING. KARL-THOMAS HÜBNER \\ TECHNISCHE UNIVERSITÄT MÜNCHEN, LEHRSTUHL FÜR FÖRDERTECHNIK MATERIALFLUSS UND LOGISTIK
}

In this paper a superelement formulation for geometric nonlinear finite element analysis is proposed. The element formulation is based on matrices generated by the static condensation algorithm. After defining the element characteristics, a method for the calculation of the element forces in a large displacement and rotation analysis is developed. In order to use the element in the solution of stability problems, the formulation of the geometric stiffness matrix is derived. An example shows the benefits of the element for the calculation of lattice-boom cranes.

\section{Introduction}

Lattice-boom cranes are tall, slender structures with acute-angled suspensions. They show a strong nonlinear behaviour near their load limits. In practice the analysis of these cranes is done quasistatic as specified in DIN 15018 T3 [DIN84] and DIN EN 13000 [DIN04] respectively.

The quasistatic analysis method represents the dynamic loading only by approximation. For this reason the European standards allow dynamic analysis methods as well: "Alternatively advanced and recognized theoretical or experimental methods (e.g. elastokinetic analysis for the simulation of load effects) ... may be used in general." [DIN04].

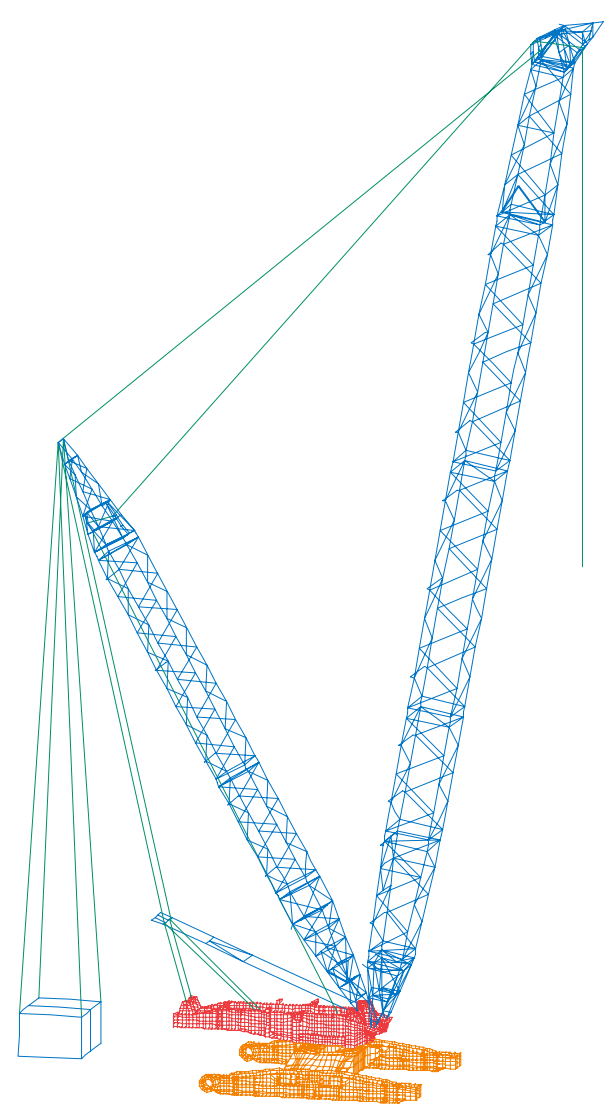

Figure 1: Finite element model of a lattice-boom crane 
Figure 1 shows an example of a finite element model of a lattice-boom crane. The beam model of the latticebooms consists of 200 to 2000 nodes, depending on the boom configuration. In contrast to this relatively small model part, the shell models of the superstructure and the carrier have up to 40000 nodes [Kleeberger96, Günthner97].

Lattice-boom cranes are built according to the modular design principle. This allows a variety of boom lengths, boom combinations and boom positions. The calculation of all lifting capacities requires up to 1000000 program runs [Günthner00-1, Günthner00-2, Webhofer05]. By using model sizes as mentioned above this cannot be done in a reasonable amount of time. So the model size must be reduced. In industrial practice the boom sections are mapped to single beam elements. The superstructure and carrier stiffness is represented by springs.

A more accurate reduction technique is static condensation. It is suitable for linear systems, such as the very stiff superstructure and carrier models. But this technique cannot be used directly for the calculation of the geometric nonlinear boom models. However, if looked on separately every single boom section fulfils small displacement conditions. So the intention is to develop a superelement formulation for geometric nonlinear finite element analysis. The element should be based on matrices generated by the static condensation algorithm.

\section{Static condensation algorithm}

The basic idea of static condensation [Bathe96, Szilard82] is to reduce the number of nodes of a finite element model part by condensing out all inner nodes. Only nodes connecting the model part to other model parts are retained. Figure 2 shows the connection nodes of a lattice-boom section model.

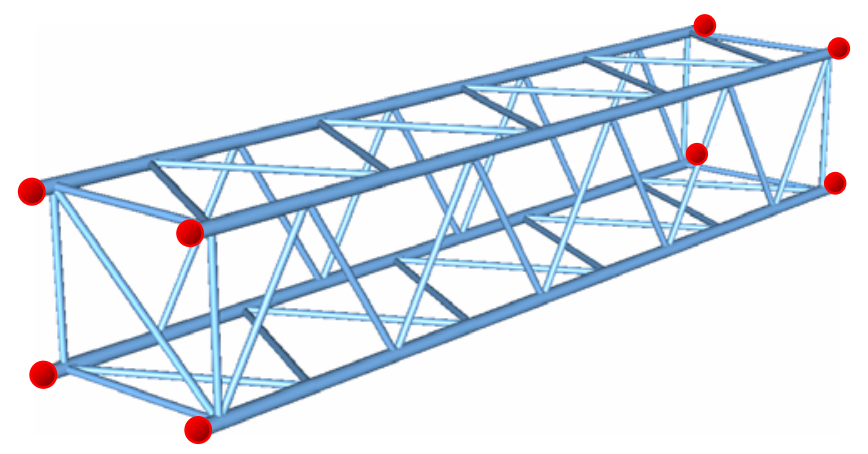

Figure 2: Connection nodes of a lattice-boom section model

The static condensation method applies to linear systems. In order to establish the equations, we assume that the stiffness matrix and corresponding displacement and force vectors are partitioned into the form

$$
\left(\begin{array}{c|c}
K_{i i} & K_{i c} \\
---- & ---- \\
K_{c i} & K_{c c}
\end{array}\right) \cdot\left(\begin{array}{c}
u_{i} \\
-- \\
u_{c}
\end{array}\right)=\left(\begin{array}{c}
R_{i} \\
-- \\
R_{c}
\end{array}\right)
$$

where $u_{i}$ and $u_{c}$ are the vectors of displacements to be retained and condensed out, respectively. The matrices $K_{i i}$, $K_{i c}$ and $K_{c c}$ and the vectors of external forces $R_{i}$ and $R_{c}$ correspond to the displacement vectors $u_{i}$ and $u_{c}$.

Using the second matrix equation in (1), we obtain:

$$
\underline{u}_{c}=K_{c c}^{-1} \cdot\left(\underline{R}_{c}-K_{c i} \cdot \underline{u}_{i}\right)
$$

Equation (2) is used to substitute for $u_{c}$ into the first matrix equation in (1) to obtain the condensed equations

$$
K^{*} \cdot \underline{u}_{i}=\underline{R}_{i}-K_{i c} \cdot K_{c c}^{-1} \cdot \underline{R}_{c}
$$

with

$$
K^{*}=K_{i i}-K_{i c} \cdot K_{c c}^{-1} \cdot K_{c i}
$$


The condensed stiffness matrix $K^{*}$ is used in the analysis of the overall model. In subsequent program runs the displacement vector $u_{c}$ and so the element forces and stresses can be evaluated for all condensed model parts. For linear systems no loss of accuracy occurs.

\section{Nonlinear equation of motion}

In contrast to a linear analysis, a nonlinear analysis must be performed by incremental steps. Equation (5) shows the transition from a known state at time $t$ to the state at time $t+\Delta t$. In general equilibrium iterations must be performed at time $t+\Delta t$ [Bathe96].

$$
\begin{gathered}
M \cdot{ }^{t+\Delta t} \underline{\ddot{u}}+D \cdot{ }^{t+\Delta t} \underline{\dot{u}}+{ }^{t} K \cdot \underline{u}={ }^{t+\Delta t} \underline{R}-{ }^{t} \underline{F} \\
{ }^{t+\Delta t} \underline{u}={ }^{t} \underline{u}+\underline{u}
\end{gathered}
$$

with

$$
\begin{array}{ll}
M: & \text { mass matrix } \\
D: & \text { damping matrix } \\
{ }^{t} K: & \text { tangent stiffness matrix at time } t \\
{ }^{t+\Delta t} R: & \text { vector of external loads at time } t+\Delta t \\
{ }^{t} F: & \text { vector of element forces at time } t \\
{ }^{t+\Delta} t & \text { vector of displacements at time } t+\Delta t \\
{ }^{t} \underline{u}: & \text { vector of displacements at time } t \\
\underline{u}: & \text { vector of incremental displacement }
\end{array}
$$

The tangent stiffness matrix ${ }^{t} K$ consists of the linear stiffness matrix ${ }^{t} K_{L}$ and the geometric stiffness matrix ${ }^{t} K_{N L}$ :

$$
{ }^{t} K={ }^{t} K_{L}+{ }^{t} K_{N L}
$$

The linear stiffness matrix is determined by the material and dimensions of the structure. The geometric stiffness matrix takes into account the effects of stresses on the structure's stability.

\section{Superelement formulation for geometric nonlinear analysis}

Based on the matrices obtained by static condensation, a superelement formulation is developed for static and dynamic geometric nonlinear analysis. The element has the following fundamental features:

- $\quad$ The element has at least three nodes; the total number of nodes $\mathrm{N}$ is unlimited

- Each element node has three translational and three rotational degrees of freedom

- $\quad$ Large displacements and rotations are allowed

- $\quad$ The element deformation remains small

- $\quad$ The element must be suitable for the solution of stability problems. I.e. the geometric stiffness matrix formulation is developed

\subsection{Positioning problem}

Figure 3 shows as an example a four node superelement in its initial state at time 0 and in its state at time $t$. The positions of the nodal points are given by the vector

$$
\underline{x}=\left(\begin{array}{c}
\underline{x}^{(1)} \\
\vdots \\
\underline{x}^{(N)}
\end{array}\right), \quad \underline{x}^{(i)}=\left(\begin{array}{c}
x \\
y \\
z
\end{array}\right)
$$

respective to the global coordinate system. 


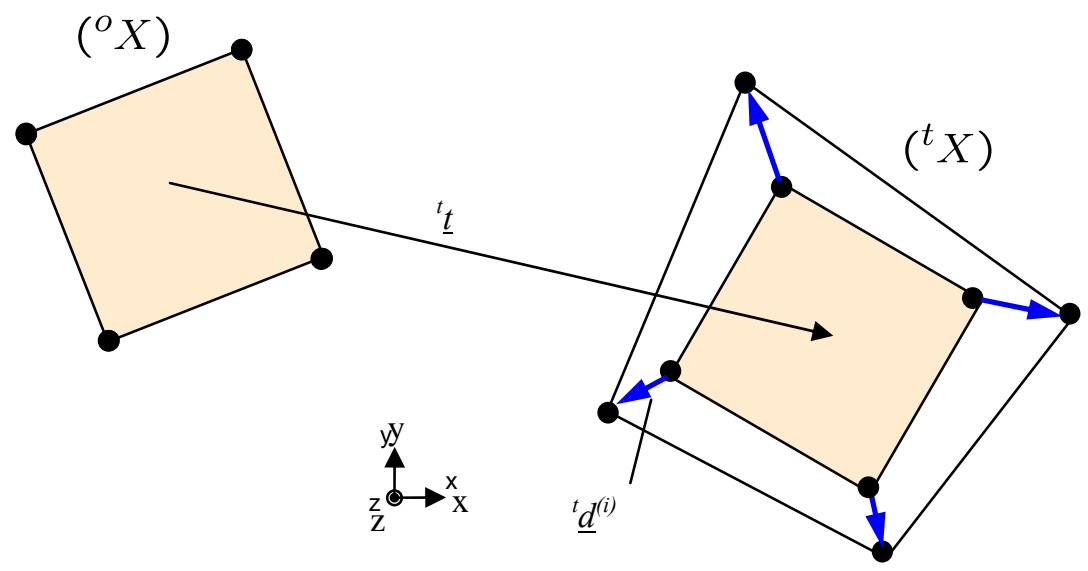

Figure 3: Initial state and state at time $t$

Since the element can undergo large displacements and rotations, we have to separate the rigid body motion from the element deformation. The position vector at time $t$ of the node $i$ is given by

$$
{ }^{t} \underline{x}^{(i)}=\underbrace{{ }^{t} \underline{t}+{ }^{t} T^{T} \cdot{ }^{o} \underline{x}^{(i)}}_{\text {rigidbody motion }}+\underbrace{{ }^{t} \underline{d}^{(i)}}_{\text {deformation }}
$$

with

$$
\begin{aligned}
& { }^{t} \underline{t} \text { : rigid body translation vector } \\
& { }^{t} T \text { : rotary matrix }
\end{aligned}
$$

The rigid body motion should put the node positions of the originally shaped element as near as possible to their positions at time $t$. One way to fulfill this condition is to minimize the displacement vector ${ }^{t} \underline{d}$, which denotes the deformation of the element:

$$
\left|{ }^{t} \underline{d}\right|^{2}=\sum_{i=1}^{N}\left|{ }^{t} \underline{x}^{(i)}-\left({ }^{t} \underline{t}+{ }^{t} T^{T} \cdot{ }^{o} \underline{x}^{(i)}\right)\right|^{2} \rightarrow \operatorname{Min}
$$

By referencing ${ }^{t} \underline{x}$ and ${ }^{0} \underline{x}$ to the center of the element, equation (9) can be transformed into the following problem of maximization [Nüchter02, Horn87]:

$$
\sum_{i=1}^{N}\left|{ }^{t} \underline{x}^{(i)^{T}} \cdot{ }^{t} T^{T} \cdot{ }^{o} \underline{x}^{(i)}\right|^{2} \rightarrow \text { Max }
$$

The rotary matrix $T$ is expressed by the unit quaternion $q$ [Blaschke60]:

$$
\begin{gathered}
q_{1}=\cos (\varphi / 2) \\
q_{2}=n_{x} \cdot \sin (\varphi / 2) \\
q_{3}=n_{y} \cdot \sin (\varphi / 2) \\
q_{4}=n_{z} \cdot \sin (\varphi / 2) \\
T=\left(\begin{array}{ccc}
q_{1}^{2}+q_{2}^{2}-q_{3}^{2}-q_{4}^{2} & 2\left(q_{2} q_{3}+q_{4} q_{1}\right) & 2\left(q_{2} q_{4}-q_{3} q_{1}\right) \\
2\left(q_{2} q_{3}-q_{4} q_{1}\right) & q_{1}^{2}-q_{2}^{2}+q_{3}^{2}-q_{4}^{2} & 2\left(q_{3} q_{4}+q_{2} q_{1}\right) \\
2\left(q_{2} q_{4}+q_{3} q_{1}\right) & 2\left(q_{3} q_{4}-q_{2} q_{1}\right) & q_{1}^{2}-q_{2}^{2}-q_{3}^{2}+q_{4}^{2}
\end{array}\right)
\end{gathered}
$$

Using the quaternion $q$ equation (10) can be transformed to

$$
{ }^{t} \underline{q}^{T} \cdot N \cdot{ }^{t} \underline{q} \rightarrow \operatorname{Max}
$$


with

$$
\begin{aligned}
& N=\left(\begin{array}{cccc}
S_{11}+S_{22}+S_{33} & S_{23}-S_{32} & S_{31}-S_{13} & S_{12}-S_{21} \\
S_{23}-S_{32} & S_{11}-S_{22}-S_{33} & S_{12}+S_{21} & S_{13}+S_{31} \\
S_{31}-S_{13} & S_{12}+S_{21} & S_{22}-S_{11}-S_{33} & S_{23}+S_{32} \\
S_{12}-S_{21} & S_{13}+S_{31} & S_{23}+S_{32} & S_{33}-S_{11}-S_{22}
\end{array}\right) \\
& S_{11}=\sum_{i=1}^{N}\left({ }^{o} x \cdot{ }^{t} x\right)^{(i)} \quad, \quad S_{22}=\sum_{i=1}^{N}\left({ }^{o} y \cdot{ }^{t} y\right)^{(i)} \quad, \quad S_{33}=\sum_{i=1}^{N}\left({ }^{o} z \cdot{ }^{t} z\right)^{(i)} \\
& S_{12}=\sum_{i=1}^{N}\left({ }^{o} x \cdot{ }^{t} y\right)^{(i)} \quad, \quad S_{21}=\sum_{i=1}^{N}\left({ }^{o} y \cdot{ }^{t} x\right)^{(i)} \quad, \quad S_{23}=\sum_{i=1}^{N}\left({ }^{o} y \cdot{ }^{t} z\right)^{(i)} \\
& S_{31}=\sum_{i=1}^{N}\left({ }^{o} z \cdot{ }^{t} x\right)^{(i)} \quad, \quad S_{32}=\sum_{i=1}^{N}\left({ }^{o} z \cdot{ }^{t} y\right)^{(i)} \quad, \quad S_{13}=\sum_{i=1}^{N}\left({ }^{o} x \cdot{ }^{t} z\right)^{(i)}
\end{aligned}
$$

According to [Horn87] the required quaternion ${ }^{t} q$ is the eigenvector corresponding to the greatest eigenvalue of the following eigenproblem:

$$
(N-\lambda I) \cdot{ }^{t} \underline{q}=\underline{0}
$$

The solution of equation (13) yields the rotary matrix ${ }^{t} T(q)$. The element deformation in the local coordinate system is given by the displacement vector ${ }^{\mathbf{t}} \underline{d}_{\underline{L}}$ :

$$
{ }^{t} \underline{d}_{L}^{(i)}={ }^{t} T(\underline{q}) \cdot{ }^{t} \underline{x}^{(i)}-{ }^{o} \underline{x}^{(i)}
$$

\subsection{Local rotations}

In order to obtain the local rotations of the element nodes a quaternion $q_{N}$ is assigned to each node. In the initial state at time $t=0$ all quaternions $\underline{q}_{N}$ are set equal to the quaternion ${ }^{0} q$ which defines the orientation of the local coordinate system. The quaternions ${ }^{t} q_{N}$ at time $t$ only depend on the solution of the equation of motion (5).

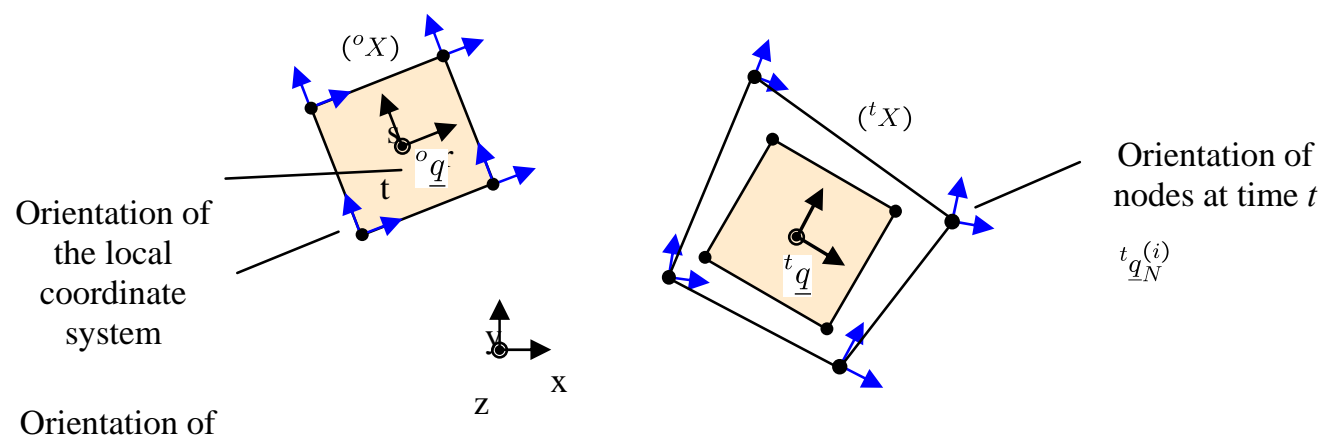

Orientation of

$$
{ }^{o} \underline{q}_{N}^{(i)}={ }^{o} \underline{q}
$$

Figure 4: Orientation of nodes 
The element deformation caused by the rotation of nodes at time $t$ is the difference between the rotations given by the quaternions ${ }^{t} \underline{q}_{N}$ and the quaternion ${ }^{t} \underline{q}$, which defines the orientation of the local coordinate system at time $t$ :

$$
\underline{q}_{\text {Diff }}^{(i)}=\left(\begin{array}{cccc}
{ }^{t} q_{N 1} & -{ }^{t} q_{N 2} & -{ }^{t} q_{N 3} & -{ }^{t} q_{N 4} \\
{ }^{t} q_{N 2} & { }^{t} q_{N 1} & -{ }^{t} q_{N 4} & { }^{t} q_{N 3} \\
{ }^{t} q_{N 3} & { }^{t} q_{N 4} & { }^{t} q_{N 1} & -{ }^{t} q_{N 2} \\
{ }^{t} q_{N 4} & -{ }^{t} q_{N 3} & { }^{t} q_{N 2} & { }^{t} q_{N 1}
\end{array}\right) \cdot\left(\begin{array}{c}
{ }^{t} q_{1} \\
{ }^{t} q_{2} \\
{ }^{t} q_{3} \\
{ }^{t} q_{4}
\end{array}\right)
$$

Provided that the element deformation remains small, equation (15) can be linearized:

$$
\underline{\varphi}^{t}=\left(\begin{array}{c}
{ }^{t} \varphi_{1} \\
{ }^{t} \varphi_{2} \\
{ }^{t} \varphi_{3}
\end{array}\right)^{(i)} \approx 2 \cdot\left(\begin{array}{c}
{ }^{t} q_{2, \text { Diff }} \\
{ }^{t} q_{3, \text { Diff }} \\
{ }^{t} q_{4, \text { Diff }}
\end{array}\right)^{(i)}
$$

\subsection{Calculation of element forces}

The element forces ${ }^{t} \underline{F}_{L}$ in local coordinates are determined by the local displacements and rotations ${ }^{t} \underline{u}_{L}$ and the stiffness matrix $K^{*}$ :

$$
{ }^{t} \underline{F}_{L}=K^{*} \cdot \underline{u}_{L}
$$

with

$$
\begin{gathered}
{ }^{t} \underline{u}_{L}=\left(\begin{array}{c}
{ }^{t} \underline{u}_{L}^{(1)} \\
\vdots \\
{ }^{t} \underline{u}_{L}^{(N)}
\end{array}\right) ; \quad{ }^{t} \underline{F}_{L}=\left(\begin{array}{c}
{ }^{t} \underline{F}_{L}^{(1)} \\
\vdots \\
{ }^{t} \underline{F}_{L}^{(N)}
\end{array}\right) \\
\underline{u}_{L}{ }^{(i)}=\left(\begin{array}{c}
{ }^{t} \underline{d}_{L}^{(i)} \\
--- \\
{ }^{t} \underline{\varphi}^{(i)}
\end{array}\right)=\left(\begin{array}{c}
{ }^{t} T(q) \cdot{ }^{t} \underline{x}^{(i)}-{ }^{o} \underline{x}^{(i)} \\
---------- \\
{ }^{t} \underline{\varphi}^{(i)}
\end{array}\right)
\end{gathered}
$$

The element forces respective to the global coordinate system are given by

$$
{ }^{t} \underline{F}={ }^{t} \hat{T}^{T} \cdot \underline{F}_{L}
$$

with

$$
{ }^{t} \hat{T}=\operatorname{diag}\left({ }^{t} T\right)_{2 N}
$$

\subsection{Superelement stiffness matrix}

The tangent stiffness used in equation (5) corresponds to the derivative of the global element forces ${ }^{t} \underline{F}$ with respect to the nodal point displacements ${ }^{t} \underline{u}$ [Bathe96]:

$$
{ }^{t} K=\frac{\partial^{t} \underline{F}}{\partial^{t} \underline{u}}
$$

Substituting the superelement forces defined in (17) into (18) yields:

$$
{ }^{t} K=\underbrace{{ }^{t} \hat{T}^{T} \cdot K^{*} \cdot{ }^{t} \hat{T}}_{K_{L}}+\underbrace{\frac{\partial{ }^{t} \hat{T}^{T}}{\partial^{t} \underline{u}} \cdot{ }^{t} \underline{F}_{L}+\left(\frac{\partial{ }^{t} \hat{T}}{\partial^{t} \underline{u}} \cdot{ }^{t} \underline{F}_{L}\right)^{T}}_{K_{N L}}
$$

The first part of (20) is the linear element stiffness matrix $K^{*}$ transformed to the global coordinate system. The second part only depends on the element position and the element forces; this is the geometric stiffness matrix. 
The rotary matrix ${ }^{t} T$ is determined by displacements, not rotations. Since the variation of ${ }^{t} \underline{u}$ corresponds to the variation of ${ }^{t} \underline{x}$, the derivatives of the components of ${ }^{t} T$ given in (11) are:

$$
\begin{gathered}
\frac{\partial{ }^{t} T_{11}}{\partial^{t} \underline{u}}=\frac{\partial{ }^{t} T_{11}}{\partial{ }^{t} \underline{x}}=-4\left(q_{4} \cdot \frac{\partial q_{4}}{\partial{ }^{t} \underline{x}}+q_{3} \cdot \frac{\partial q_{3}}{\partial{ }^{t} \underline{x}}\right) \\
\frac{\partial^{t} T_{21}}{\partial^{t} \underline{u}}=\frac{{ }^{t} T_{21}}{\partial^{t} \underline{x}}=2\left(q_{2} \cdot \frac{\partial q_{3}}{\partial^{t} \underline{x}}+q_{3} \cdot \frac{\partial q_{2}}{\partial^{t}{ }^{t} \underline{x}}-q_{4} \cdot \frac{\partial q_{1}}{\partial{ }^{t} \underline{x}}-q_{1} \cdot \frac{\partial q_{4}}{\partial{ }^{t} \underline{x}}\right)
\end{gathered}
$$

Differentiating equation (5) on side condition that ${ }^{t} q$ is a unit vector yields:

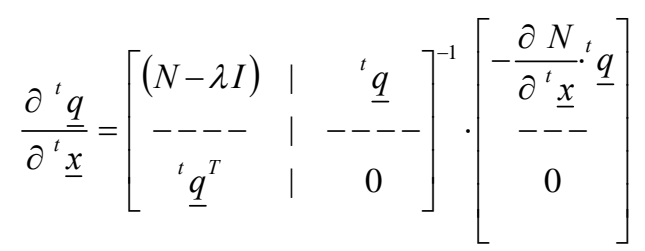

By substituting equation (22) into (21) the geometric stiffness matrix can be evaluated.

\section{Example of use}

The superelement formulation was implemented in the finite element program NODYA [Kleeberger04]. Figure 5 shows the model of the lattice boom crane used for testing. It consists of the carrier, superstructure and main boom. The model generation, static condensation and postprocessing was done using I-DEAS [Unigraphics04].

In order to compare a conventional finite element model with the superelement formulation, a nonlinear static analysis of load case $\mathrm{H}$ (gravitational forces, hoisting and slewing forces) was performed by NODYA.

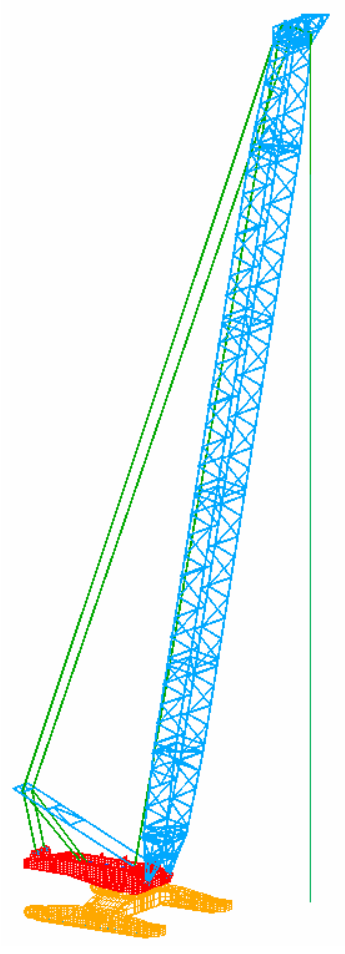

Figure 5: Test system

Table 1 shows the number of degrees of freedom of the original and condensed model parts. The best reduction rate is achieved for the carrier and superstructure. The overall reduction is from 69162 degrees of freedom to 798. 
Table 1: structural data

\begin{tabular}{|l|l|l|}
\hline--- & original model & superelement \\
\hline carrier & 43740 & 252 \\
\hline superstructure & 23028 & 222 \\
\hline foot section & 246 & 36 \\
\hline boom extension & 222 & 48 \\
\hline boom section 6m & 288 & 48 \\
\hline boom section 12m & 402 & 48 \\
\hline adapter top section & 222 & 48 \\
\hline top section & 330 & 60 \\
\hline
\end{tabular}

The comparison is made between the original boom model with condensed carrier and superstructure and the overall superelement model. Figure 6 shows the results for two different main boom angles.

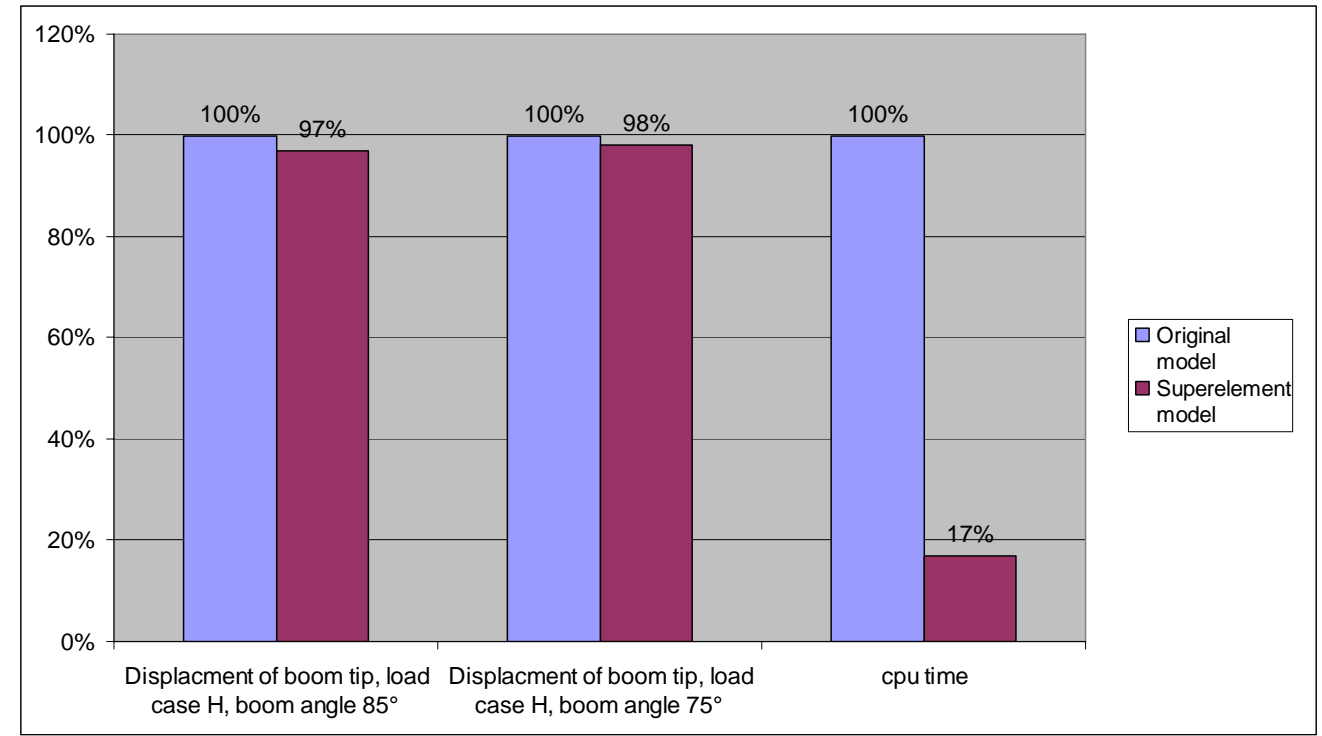

Figure 6: Example results

The results show a minimal loss of accuracy and a significant reduction of cpu-time.

Further tests concerning the behaviour of the superelement in dynamic analysis and a comparison of the results with multi-body simulation programs (e.g. Adams [Adams05]) is planned.

\section{Conclusion}

The overall calculation of load capacities of lattice-boom cranes requires small finite element models. The superelement presented in this paper offers a sophisticated reduction technique that meets the requirements of geometric nonlinear analysis. The element significantly reduces the calculation time and causes a negligible loss of accuracy. The direct coupling of reduced and original models insures the consistency and allows the evaluation of forces and stresses in subsequent program runs. 


\section{7. $\quad$ Literature}

[Adams05] MSC Software Corporation; MSC.ADAMS 2005 r2 - Installation and Operations Guide : Santa Ana, 2005

[Bathe96] Bathe, K.-J.: Finite Element Procedures : Prentice Hall, 1996

[Blaschke60] Blaschke, W.: Kinematik und Quaternionen. Berlin : VEB Deutscher Verlag der Wissenschaften, 1960

[DIN84] Norm DIN 15018-3:1984-11: Cranes, principles for steel structures, stress analysis of mobile cranes, German Version

[DIN04] Norm DIN EN 13000:2004-09 : Cranes - Mobile cranes; German version

[Günthner97] Günthner, W.A. ; Kleeberger, M.: Zum Stand der Berechnung von GittermastFahrzeugkranen. In:Deutsche Hebe- und Fördertechnik, dhf 43 (1997) Nr. 3, S. 56-61

[Günthner00-1] Günthner, W.A. ; Schröder, F.: Automatisierte Modellgenerierung zur Berechnung von Gittermastkranen - Teil I. In: Fördern und Heben: f+h 50 (2000) 7, S. 506-507

[Günthner00-2] Günthner, W.A. ; Schröder, F.: Automatisierte Modellgenerierung zur Berechnung von Gittermastkranen - Teil II. In: Fördern und Heben: f+h 50 (2000) 8, S. 587-589

[Horn87] Horn, B.: Closed-form solution of absolute orientation using unit quaternions. In: Journal of the Optical Society of America 4 (1987), S. 629-642

[Kleeberger04] Kleeberger, M.: NODYA Version 1.1, Benutzeranweisung. Lehrstuhl für Fördertechnik Materialfluss Logistik, Technische Universität München, 2004

[Kleeberger96] Kleeberger, M.: Nichtlineare dynamische Berechnung von Gittermast-Fahrzeugkranen. München, Technische Universität, Diss., 1996

[Nüchter02] Nüchter, A.: Autonome Exploration und Modellierung von 3D-Umgebungen. Fraunhofer Institut für Autonome Intelligente Systeme, Institut für Informatik III Volltext s. http://www.ais.fraunhofer.de/ARC/3D/scanner/cdindex.html

[Unigraphics04] Unigraphics Solutions Inc.: I-DEAS User's Guide, Cypress, 2004

[Szilard82] Szilard, R.: Finite Berechnungsmethoden der Strukturmechanik, Bd. 1. Berlin u.a.: Verlag von Wilhelm Ernst \& Sohn, 1982

[Webhofer05] Webhofer M.: Verwendung kinematischer Ketten bei der Generierung von Finite-ElementeModellen, In: Logistics Journal : Nicht-referierte Veröffentlichungen, Januar 2005. http://www.elogistics-journal.de/uploads/media/Kinematische_Ketten.pdf, DOI: $10.2195 /$ LJ_Not_Ref_d_Webhofer_0120051 urn:nbn:de:0009-14-7675 